\title{
MarcoPolo-R: Near Earth Asteroid Sample Return Mission candidate as ESA-M3 class mission
}

\author{
Patrick Michel \\ and the MarcoPolo-R Science Study Team
}

Laboratoire Lagrange, University of Nice Sophia-Antipolis, Côte d'Azur Observatrory

BP 4229, 06304 Nice Cedex 4, France

email: michelp@oca.eu

\begin{abstract}
MarcoPolo- $R$ is a sample return mission to a primitive Near-Earth Asteroid (NEA) selected in 2011 for the Assessment Study Phase of M3-class missions in the framework of ESAs Cosmic Vision (CV) 2015-2025 programme. The phase A study started at the end of 2012 and will proceed throughout 2013. The final selection by ESA will occur in February 2014. MarcoPolo- $R$ is a European-led mission with a possible contribution from other agencies. MarcoPolo- $R$ will rendez-vous with the primitive NEA 2008 EV5. Before returning a unique sample to Earth, the asteroid will be scientifically characterized at multiple scales. MarcoPolo- $R$ will provide detailed knowledge of the physical and compositional properties of a member of the population of Potentially Hazardous Asteroids (PHA), which is an important contribution to mitigation studies.
\end{abstract}

Keywords. celestial mechanics, space vehicles: instruments, minor planets, asteroids. 